\title{
BWL und VWL für Juristen: Ergebnisse einer Bildungsbedarfsanalyse im Studiengang Wirtschaftsrecht
}

\author{
Julia Riha*
}

\begin{abstract}
Eine Bildungsbedarfsanalyse dient als Grundlage zur Gestaltung von Studienangeboten und ermittelt möglichst genau und systematisch, was in einem Studiengang, einem Modul oder in einer einzelnen Veranstaltung überhaupt gelernt werden soll. ${ }^{1}$ Konkretes Ziel der Bildungsbedarfsanalyse im Studiengang Wirtschaftsrecht war die Überprüfung von Breite und Tiefe des Angebots an wirtschaftswissenschaftlichen Veranstaltungen als Grundlage der Curriculumentwicklung. Die Bildungsbedarfsanalyse wurde in Form einer telefonischen Absolventenbefragung durchgeführt. Die Absolventen ${ }^{2}$ wurden insbesondere zu Anwendungsmöglichkeiten wirtschaftswissenschaftlicher Studieninhalte in ihrer aktuellen Tätigkeit sowie rückblickend zum Angebot betriebs- und volkswirtschaftlicher Inhalte im Studiengang befragt. Im Ergebnis zeichnen sich ein heterogener Anspruch an die wirtschaftswissenschaftlichen Lehrveranstaltungen und ein unterschiedlich großer Anwendungsbezug der wirtschaftswissenschaftlichen Inhalte im Berufsleben ab. Die meisten Studierenden möchten durch die Zusatzqualifikation in erster Linie ein Verständnis für die wirtschaftswissenschaftliche Denkweise und Fachsprache erlangen.
\end{abstract}

\section{A. Bildungsbedarfsanalyse durch Absolventenbefragung}

Was sollten Wirtschaftsjuristen in Betriebswirtschaftslehre (BWL) und in Volkswirtschaftslehre (VWL) können? Diese Fragestellung war ausschlaggebend für die Durchführung der Bildungsbedarfsanalyse, denn in der wirtschaftsjuristischen Weiterbildung wird an der Universität zu Köln neben der Vertiefung von Rechtsgebieten betriebs- und volkswirtschaftliches Grundwissen erworben. Eine Bildungsbedarfsanalyse in Form einer Absolventenbefragung stellt dabei eine Möglichkeit dar, das Lehrangebot stärker an den Bedürfnissen der Teilnehmenden am Studiengang auszurichten und möglichst genau und systematisch zu ermitteln, was in einem Studiengang, einem Modul oder in einer einzelnen Veranstaltung überhaupt gelernt werden soll. ${ }^{3}$

Der Studiengang Wirtschaftsrecht an der Universität zu Köln ist berufsbegleitend. Er richtet sich an Referendare, Doktoranden, Assessoren, Rechtsanwälte sowie Unternehmensjuristen, die neben der Vertiefung von Rechtsgebieten auch wirtschaftswis-

* Die Autorin ist wissenschaftliche Mitarbeiterin am Kompetenzzentrum für juristisches Lernen und Lehren der Universität zu Köln.

1 Müller, in: Schweizer/Iberer et al. (Hrsg.), S. 99 (114).

2 Zur besseren Lesbarkeit wird im folgenden Text das generische Maskulinum verwendet. Gleichermaßen sind jedoch Frauen und Männer angesprochen.

3 Müller, in: Schweizer/Iberer et al. (Hrsg.), S. 99 (114). 
senschaftliche Kenntnisse erwerben wollen. Er ist als Masterstudiengang konzipiert und schließt mit dem Erwerb des „Master of Laws“ (LL.M.) ab.

Mithilfe der Bildungsbedarfsanalyse sollte herausgefunden werden, welche betriebsund volkswirtschaftlichen Kenntnisse bzw. Kompetenzen die Absolventen des Studienganges in ihrem Berufsalltag nutzen können. Die Ergebnisse dienen der curricularen Weiterentwicklung im Studiengang, um das Lehrangebot besser an den Bedürfnissen der Zielgruppe auszurichten. Hierbei wurde einerseits die Breite, d.h. das Angebot an unterschiedlichen wirtschaftswissenschaftlichen Lehrveranstaltungen, andererseits die Tiefe, d.h. die Vertiefung einzelner Fachgebiete innerhalb der Wirtschaftswissenschaften, überprüft. Dieser Werkstattbericht stellt beispielhaft dar, wie anhand einer Absolventenbefragung der Bildungsbedarf erfasst, analysiert und zur Curriculumentwicklung in einem Studiengang eingesetzt werden kann.

Bei der Weiterentwicklung des Curriculums stand also die Frage im Vordergrund, welche konkreten wirtschaftswissenschaftlichen Inhalte bzw. Kompetenzen ein Wirtschaftsjurist im späteren Berufsalltag benötigt. Auch wenn im Zusammenhang mit der Kompetenzorientierung von Lehrplänen eine Diskussion um Inhalte nicht mehr im Vordergrund steht, soll hier bewusst von Lehr-/Lerninhalten die Rede sein. Diese können dann als Grundlage für eine kompetenzorientierte Lehrplangestaltung dienen.

Kompetenzen in ihrer Definition als Befähigung zur Bewältigung beruflicher Handlungssituationen ${ }^{4}$ lassen sich am besten an der Berufspraxis „handelnder Absolvent“ überprüfen. Als Methode dafür bietet sich die Bildungsbedarfsanalyse an, zielt sie doch auf eine systematische Ermittlung des Bildungsbedarfes aus Sicht der Lernenden. ${ }^{5}$

Die Art der eingangs gestellten Frage impliziert dabei bereits eine Nützlichkeitsorientierung ${ }^{6}$ und eine Ausrichtung des Gelernten an beruflich verwertbaren Inhalten. Somit ist bereits ein Relevanzkriterium ${ }^{7}$ zur Legitimation der Lehr-/Lerninhalte gesetzt. Vor dem Hintergrund allgemeiner Diskussionen über die Ausrichtung von Bildungsangeboten einer Universität sowie speziell über Lehr-/Lerninhalte der juristischen universitären Ausbildung muss jedoch darüber hinaus überlegt werden, welche

4 Der Kompetenzbegriff bzw. die Kompetenzorientierung in Studium und Lehre hat seit geraumer Zeit die Orientierung an Inhalten ergänzt, wenn nicht sogar abgelöst. Die hier zugrunde gelegte Definition greift das Kompetenzverständnis der Berufspädagogik auf und stellt die Entwicklung beruflicher Handlungskompetenz, d.h. die Befähigung für bestimmte berufliche Handlungsfelder in den Mittelpunkt. Dazu bspw. Schaper, S. 16.

5 Damit werden die sogenannten subjektiven Bildungsbedürfnisse in Erfahrung gebracht. Ein anderer Blickwinkel entstünde durch den Fokus auf objektive Bildungsbedarfe der „abnehmenden Systeme“, d.h. Bildungsbedarfe von Arbeitgebern und der Gesellschaft (dazu Müller, in: Schweizer/Iberer et al. (Hrsg.), S. 99 (114)).

6 Zur Diskussion um Nützlichkeit von Studieninhalten, insbesondere im Zuge der Bologna-Reform, siehe z.B. Teichler, in: Das Hochschulwesen 56 (3), S. 68 ff.

7 In der Curriculumentwicklung geht es darum, Kriterien für die Relevanz von Lehr-/Lernzielen und Inhalten festzulegen, mit deren Hilfe bedeutsame Ziele und Inhalte ermittelt, ausgewählt und begründet werden können. In der Curriculumtheorie werden zur Lösung des Relevanzproblems unterschiedliche Vorschläge gemacht, z.B. von Reetz, Wirtschaftsdidaktik, S. 76 ff. 
weiteren Relevanzkriterien zur Legitimation der Lehr-/Lerninhalte herangezogen werden können. ${ }^{8}$ Bevor auf das Vorgehen und die Ergebnisse der Absolventenbefragung eingegangen wird, sollen deshalb weitere mögliche Relevanzkriterien skizziert und das für die Bildungsbedarfsanalyse ausgewählte Kriterium begründet werden.

\section{B. Theoretischer Hintergrund}

Die Idee der Curriculumlegitimation richtet sich auf das Zustandekommen von Lehrplänen. Angestrebt wird eine Begründung, warum bestimmte Lehr-/Lerninhalte als legitimiert gelten können. Da es einen allgemein anerkannten Grundkonsens dieser Art nicht geben kann, muss die Auswahl der Legitimationsquellen von Fall zu Fall begründet werden. ${ }^{9}$

Zur Auswahl und Legitimation von Lerninhalten gibt es verschiedene Ansätze, die als übergeordnete Normen die Entscheidung für bestimmte Inhalte begründen. ${ }^{10} \mathrm{Die}$ folgende Klassifizierung orientiert sich an Euler/Hahn, die ihrerseits auf die Überlegungen von Reetz $z^{11}$ zurückgreifen. Die drei „Legitimationsquellen“ (so der Wortlaut bei Euler/Hahn) bzw. „Relevanzprinzipien“ (Reetz) „Situationsprinzip, Wissenschaftsprinzip und Persönlichkeitsprinzip“ lassen sich durchaus miteinander verbinden. Hierfür ist jedoch die Entscheidung wichtig, welches Prinzip den jeweils anderen über- bzw. untergeordnet sein soll. ${ }^{12}$ Wie eingangs skizziert, wird im Hinblick auf den weiterbildenden Masterstudiengang Wirtschaftsrecht die berufliche Verwertbarkeit des Gelernten in den Vordergrund gerückt.

Im Folgenden werden die Legitimationsquellen kurz vorgestellt. Anschließend wird darauf eingegangen, welche Konsequenzen sich daraus für das Vorgehen bei der Bildungsbedarfsanalyse ergeben.

\section{Lebenssituation der Lernenden als Legitimationsquelle}

Die Bewältigung von (beruflichen) Lebenssituationen der Lernenden steht bei dieser Norm im Vordergrund. Sie schafft eine enge Verbindung zwischen Theorie und Praxis. Über das Situationsprinzip werden Inhalte dahingehend legitimiert, dass sie die Studierenden auf die Bewältigung von Lebenssituationen vorbereiten. So wird nach Reetz „die Lebenswirklichkeit der Lernenden zum Bezugspunkt [...] gemacht“.${ }^{13}$

Der Weiterbildungsstudiengang Wirtschaftsrecht an der Universität zu Köln ist darauf ausgerichtet, der „Nachfrage nach Fachleuten mit Führungsqualitäten und ver-

8 So mögen manche konstatieren, eine Orientierung von Lehr-/Lerninhalten an Arbeitsmarktanforderungen negiere die Idee der Wissenschaftlichkeit universitärer Studienangebote und untergrabe die Freiheit von Forschung und Lehre.

9 Hameyer, in: Hameyer/Frey et al. (Hrsg.), S. 53 (63).

10 Euler/Hahn, Wirtschaftsdidaktik, S. 125.

11 Reetz, Wirtschaftsdidaktik, S. $86 \mathrm{f}$.

12 Euler/Hahn, Wirtschaftsdidaktik, S. 125 ff.

13 Reetz, Wirtschaftsdidaktik, S. $99 \mathrm{ff}$. 
tieften wirtschaftsrechtlichen und betriebswirtschaftlichen Kenntnissen " 14 entgegenzukommen. Die Teilnehmenden haben mindestens das erste Staatsexamen absolviert, viele belegen den Studiengang parallel zum Referendariat oder aber auch, nachdem sie bereits einige Jahre im Berufsleben standen. Das Situationsprinzip ist also durch die Art der Forschungsfrage („Was sollten Wirtschaftsjuristen in BWL und VWL können?“) sowie durch die Ausrichtung als weiterbildender, berufsbegleitender Studiengang gesetzt und wird als oberste Norm zu Legitimation der Lehr-/ Lerninhalte sowie als Ausgangspunkt für didaktisches Handeln herangezogen. ${ }^{15}$

Methodisch betrachtet können Situationsanalysen bestenfalls auf differenzierten, systematischen Untersuchungen beruhen. ${ }^{16}$ Dies soll durch die Absolventenbefragung erreicht werden.

\section{Wissenschaftliche Aussagen und Methoden als Legitimationsquelle}

Die Kernidee der Heranziehung wissenschaftlicher Aussagen und Methoden als Legitimationsquelle besteht in der Auffassung, dass Lerninhalte aus einer didaktisch reduzierten Struktur von Wissenschaft bestehen. Handlungskompetenzen werden als Verfügung über einen bestimmten Kanon an wissenschaftlichen Aussagen und Methoden definiert. ${ }^{17}$ Durch die Verwissenschaftlichung der Arbeits- und Lebensbedingungen und durch die Begünstigung des Lernens durch Wissenschaft werden wissenschaftsorientierte Curriculumansätze legitimiert. ${ }^{18}$

In einem zweiten Schritt geht es in den wirtschaftswissenschaftlichen Veranstaltungen darum, die für die Lebenssituationen relevanten betriebs- und volkswirtschaftlichen wissenschaftlichen Aussagen zu identifizieren und aufzubereiten. Diese Aufgabe obliegt den jeweils Lehrenden, so dass mithilfe der Absolventenbefragung keine Aussagen zur wissenschaftlichen Aufbereitung der Lehr-/Lerninhalte getroffen werden können.

\section{III. (Bildungs-)Idealvorstellung über die Persönlichkeit des Lernenden als Legitimationsquelle}

Das Persönlichkeitsprinzip betont die Entwicklung individueller Ansprüche vor sozialen Anforderungen. ${ }^{19}$ Es werden Lehr-/Lerninhalte ausgewählt, die in besonderer Weise an den Bedürfnissen des Individuums und seiner Persönlichkeitsentwicklung orientiert sind. Damit wird ein wichtiges Regulativ in die Diskussion um die Legiti-

14 Siehe Internetseite des Studiengangs: http://www.jura.uni-koeln.de/index.php?id=1081 (13.12.2013).

15 Die Ausrichtung am Situationsprinzip wird später durch die Ergebnisse der Absolventenbefragung bestätigt.

16 Euler/Hahn, Wirtschaftsdidaktik, S. 130.

17 Euler/Hahn, Wirtschaftsdidaktik, S. 126.

18 Reetz, Wirtschaftsdidaktik, S. $86 \mathrm{f}$.

19 Euler/Hahn, Wirtschaftsdidaktik, S. 128. 
mation von Lehr-/Lerninhalten eingebracht, damit didaktisches Handeln auf die individuelle Persönlichkeit der oder des Lernenden gerichtet ist. ${ }^{20}$

Die Anpassung an die individuellen Ansprüche der Lernenden erfolgt in einem dritten Schritt immer wieder neu im Rahmen der Veranstaltungen selbst, da jedes Semester Teilnehmer mit unterschiedlichen Erwartungen, Vorkenntnissen und Bedürfnissen die wirtschaftswissenschaftlichen Lehrveranstaltungen besuchen. Bei der Anpassung der wissenschaftlich aufbereiteten Lebenssituationen an die jeweilige Gruppe sind erneut die Lehrenden gefragt.

Die Bildungsbedarfsanalyse stützt sich somit lediglich auf eines der Relevanzkriterien, denn durch die Absolventenbefragung können berufliche Anforderungssituationen erfasst werden und in die Lehrplangestaltung einfließen. Informationen zur situativen Nutzung von Lehr-/Lerninhalten im beruflichen Alltag lassen sich am besten bei ehemaligen Studierenden erfragen. Es ist danach Aufgabe der Lehrenden, das Wissenschafts- sowie das Persönlichkeitsprinzip durch die konkrete Ausgestaltung der Lehrveranstaltungen zu berücksichtigen.

\section{Forschungsdesign}

Die Absolventenbefragung wurde im Zeitraum von Oktober 2012 bis Januar 2013 durchgeführt. Alle Absolventen der Jahrgänge 2003 bis 2011 ( $n=283$ ) wurden mit einer individualisierten E-Mail kontaktiert und um ein Telefoninterview gebeten. 34 E-Mail Adressen erwiesen sich als ungültig, 249 E-Mails wurden erfolgreich versandt. Davon wiederum antworteten 214 Angeschriebene nicht, 35 hingegen erklärten sich zum Interview bereit. Eine Ausweitung der Stichprobe war nicht möglich, da sämtliche zum Befragungszeitpunkt ehemalige Teilnehmer des Studiengangs angeschrieben wurden. Mit insgesamt 27 Ehemaligen kam ein Telefoninterview zustande. Die Interviews dauerten zwischen 10 und 30 Minuten und basierten auf einem Leitfadengespräch. Diese Form der offenen Gesprächsführung zielte darauf ab, einen breiten Antwortspielraum der Befragten zuzulassen und diese zu umfassenden Antworten zu bewegen, ${ }^{21}$ um so möglichst viel über die Nutzung wirtschaftswissenschaftlicher Inhalte in ihrer derzeitigen beruflichen Tätigkeit, aber auch über ihre Erfahrungen mit den wirtschaftswissenschaftlichen Kursen im Studiengang Wirtschaftsrecht herauszufinden.

Mithilfe dieser Methode lassen sich Einblicke in die Erfahrungshintergründe der Befragten erlangen. Dabei wird die Befragung auf Basis eines Leitfadens geführt, so dass zumindest eine rudimentäre Vergleichbarkeit der Interviewergebnisse gewährleistet werden kann. Die Ausformulierung und Reihenfolge der Themenbearbeitung und Fragestellungen wird vom Interviewer geleistet, um einen möglichst natürlichen Interaktionsfluss zu erreichen. ${ }^{22}$

20 Reetz, Wirtschaftsdidaktik, S. 98.

21 Schnell/Hill et al., Methoden der empirischen Sozialforschung, S. 386.

22 Schnell/Hill et al., Methoden der empirischen Sozialforschung, S. $386 \mathrm{f}$. 
Alle Interviews wurden von der Autorin selbst durchgeführt. Folgende Fragen wurden in unterschiedlicher Abfolge und bei Verständnisschwierigkeiten mit Anpassungen in der Formulierung im Laufe eines Gespräches thematisiert:

- Nennen Sie Ihren derzeitigen Arbeitgeber und Ihre Position.

- Schildern Sie kurz Ihre aktuelle Tätigkeit.

- Vor welchem Hintergrund und mit welchem Ziel hatten Sie sich zum Studium „Wirtschaftsrecht" entschieden?

- Was sollten Wirtschaftsjuristen grundsätzlich aus dem Bereich der BWL können?

- Was sollten Wirtschaftsjuristen grundsätzlich aus dem Bereich der VWL können?

- Welche dieser Kenntnisse/Kompetenzen finden sich in Ihrem Arbeitsalltag wieder?

- Wie viel Zeit innerhalb Ihrer Kernaufgaben verbringen Sie wöchentlich mit Tätigkeiten, bei denen Sie wirtschaftswissenschaftliche Kenntnisse benötigen? (weniger als 20 Prozent/zwischen 20 und 50 Prozent/mehr als 50 Prozent)

- Welche betriebs- und volkswirtschaftlichen Inhalte aus dem Studium „Wirtschaftsrecht" waren für Ihre derzeitige Tätigkeit hilfreich?

- Was hätten Sie in diesem Studiengang gerne zusätzlich für Ihre derzeitige Tätigkeit erlernt?

- Bezogen auf wirtschaftswissenschaftliche Inhalte im Masterstudiengang Wirtschaftsrecht:

- Welche Ansprüche hatten Sie an die Lehrveranstaltungen?

- Welche Ansprüche bzw. Ziele sollten sich Ihrer Meinung nach Dozenten und Studierende im Allgemeinen setzen?

Ein Gespräch wurde jeweils durch Notizen während der Befragung dokumentiert. Zusätzlich wurde direkt im Anschluss an das Telefonat ein nachträgliches Gedächtnisprotokoll angefertigt.

Die Auswertung wurde mithilfe einer quantitativen Inhaltsanalyse durchgeführt. Deren zentrale Idee besteht darin, die Vielzahl der Wörter eines Textes (hier: der Gesprächsnotizen) in Kategorien einzuordnen. Die Gesamtheit aller Kategorien wird als Kategoriensystem bezeichnet. ${ }^{23}$ Zur Auswertung wurden die Aussagen zunächst grob zu vier Themenbereichen zusammengefasst (siehe Abschnitte D. I. - D. IV. in diesem Bericht), welche die Grundlage zur Erstellung des Kategoriensystems bildeten.

Das Kategoriensystem kann vor der empirischen Erhebung (deduktives Vorgehen) oder nach der empirischen Erhebung (induktives Vorgehen) entwickelt werden. ${ }^{24}$ Hier wurde bewusst ein auf Offenheit angelegtes und somit induktives Verfahren gewählt, um ein breites Spektrum an Antwortmöglichkeiten zuzulassen ${ }^{25}$ und die Aussagen der Befragten nicht durch ein standardisiertes Interview von vornherein einzuschränken. So wurden die Gesprächsnotizen im Anschluss an die Interviews gesichtet und für eine Auszählung geeignete Kategorien festgelegt. Ausschlaggebend

23 Böhm-Kaspar/Schuchart et al., Quantitative Methoden in der Erziehungswissenschaft, S. 93.

24 Böhm-Kaspar/Schuchart et al., Quantitative Methoden in der Erziehungswissenschaft, S. 93.

25 Kromrey/Strübing, Empirische Sozialforschung, S. 25. 
ist, vom konkreten Textmaterial zu abstrahieren und zu versuchen, übergeordnete Kategorien zu finden. Diese übergeordneten Kategorien werden anhand der Textpassagen weiter in Unterkategorien unterteilt um festzulegen, welche Textmerkmale durch Auszählen erfasst werden können. ${ }^{26}$ Jeder Unterkategorie wird ein Code zugeordnet (z.B. 4.1.1, 4.1.2 usw.), der den einzelnen Aussagen der Interviewten zugeordnet werden kann, wenn sich die Aussage auf diese Unterkategorie bezieht.

Diese Codierung wurde in der Auswertung genutzt, um mithilfe der Gesprächsnotizen die Aussagen der Absolventen den einzelnen Unterkategorien zuzuordnen. Abschließend wurden diese Codes ausgezählt und mit Textbeispielen versehen. Durch das Auszählen wurde ermittelt, wie häufig die Ausprägung eines Merkmals (z.B. wirtschaftswissenschaftliche Sprache sprechen) in der Gesamtheit aller Interviews aufgetreten ist. ${ }^{27}$ Auf diese Weise wurden die absoluten bzw. relativen Häufigkeiten in den Ergebnissen ermittelt. Die Textbeispiele verdeutlichen, was in der jeweiligen Unterkategorie ausgezählt wurde. Beispielhaft ist im Folgenden ein Ausschnitt der Auswertung aufgeführt:

\begin{tabular}{|c|c|c|c|c|c|}
\hline Themenbereich & Kategorie & Unterkategorie & Anzahl & Anteil & Textbeispiele \\
\hline \multirow[t]{4}{*}{$\begin{array}{l}\text { 4. Aussagen zu hilfreichen } \\
\text { wirtschaftswissenschaftlichen } \\
\text { Kompetenzen und } \\
\text { Fachgebieten für } \\
\text { Wirtschaftsjuristen }\end{array}$} & 4.1 Kompetenzen & $\begin{array}{l}\text { 4.1.1 Wirtschafts- } \\
\text { wissenschaftliche Denkweise } \\
\text { verstehen }\end{array}$ & 13 & 0,34 & $\begin{array}{l}\text { Denken in Opportunitäten; Methodologie; } \\
\text { Ahnung haben wie die Volkswirte vorgehen; } \\
\text { Herangehensweise der VWL; Unterschied } \\
\text { BWL / VWL; wie funktioniert ein Betrieb; } \\
\text { ökonomisches Prinzip; den größeren } \\
\text { Zusammenhang wie eine Volkswirtschaft } \\
\text { funktioniert; ges amtwirtschaftliche } \\
\text { Zusammenhänge; Wirtschaftssystem; } \\
\text { anderer Blick auf das } \\
\text { Gesetzgebungsverfahren; WiWis denken } \\
\text { anders; juristische Grundannahmen müssen } \\
\text { erstmal überwunden werden }\end{array}$ \\
\hline & & $\begin{array}{l}\text { 4.1.2 Wirtschafts- } \\
\text { wissenschaftliche } \\
\text { Fachsprache sprechen }\end{array}$ & 13 & 0,34 & $\begin{array}{l}\text { Einzahlungen, Einnahmen etc.; Vokabular für } \\
\text { Gespräche mit Banken; bankspezifische } \\
\text { Terminologie; Grenznutzen; } \\
\text { Konsumentensouveränität; } \\
\text { Deckungsbeiträge; ROI; Kosten-Nutzen- } \\
\text { Verhältnisse; Begriffe wie z.B. } \\
\text { Opportunitätskosten kennen; knappe Güter; } \\
\text { richtige Fragen stellen; Überblick Vokabular }\end{array}$ \\
\hline & & $\begin{array}{l}\text { 4.1.3 Zahlen erfassen und } \\
\text { Berechnungen durchführen }\end{array}$ & 12 & 0,32 & $\begin{array}{l}\text { Einführung z.B. für Kennzahlen; Break-Even- } \\
\text { Point berechnen; Verbesserungen der } \\
\text { Rentabilität erkennen und beurteilen; } \\
\text { Auswertung von Statistiken; Kalkulationen; } \\
\text { wirtschaftliche Situation eines Unternehmens } \\
\text { einschätzen können; Zinsberechnung; } \\
\text { Grenzkostenberechnung aber dafür wäre } \\
\text { mehr Mathe erforderlich; wie ermittelt man } \\
\text { Gewinn; Mathe auffrischen wäre gut } \\
\text { gewesen; Zinsrechnung; mathematische } \\
\text { Grundlagen; Unterschied netto und brutto } \\
\text { berechnen können; Dreisatz, } \\
\text { Prozentrechnung, Bruchrechnung; konkretes } \\
\text { Problem selbst rechnen zu müssen }\end{array}$ \\
\hline & & n & 38 & & \\
\hline
\end{tabular}

Abbildung 1: Ausschnitt aus dem Kategoriensystem und der Auszählung

26 Böhm-Kaspar/Schuchart et al., Quantitative Methoden in der Erziehungswissenschaft, S. $93 \mathrm{ff}$.

27 Böhm-Kaspar/Schuchart et al., Quantitative Methoden in der Erziehungswissenschaft, S. 96. 
Neben den 27 Interviews wurden zusätzlich drei schriftliche Rückmeldungen in die Auszählung integriert. Die Grundgesamtheit in den einzelnen Kategorien unterscheidet sich, da durch die Form des Interviews nicht jeder Gesprächspartner gleichermaßen ausführlich auf die einzelnen Fragen antwortete. Manche Befragte äußerten sich zu einigen Themenbereichen gar nicht. Andere wiederum gaben mehrere Antworten, die der gleichen Unterkategorie zuzuordnen waren. Diese Mehrfachnennungen wurden in der Auszählung zugelassen und berücksichtigt.

Kritisch ist anzumerken, dass sich eine sinnvolle Bildung von Unterkategorien an manchen Stellen als schwierig erwies, wenn viele unterschiedliche Antworten gegeben wurden, die sich nicht bündeln ließen. Dies lag beispielsweise an Aussagen, die nicht nach dem Leitfaden erfragt wurden. Oftmals verfielen die Befragten „ins Erzählen “ und gaben Informationen preis, die nicht durch die eigentliche Fragestellung der Bildungsbedarfsanalyse intendiert waren. Diese Informationen wurden jedoch trotzdem in die Auswertung einbezogen, was die Vielzahl der Unterkategorien erklärt und eine sinnvolle Kategorienbildung an manchen Stellen erschwerte.

Zudem ist die Größe der Stichprobe bei einer quantitativen Inhaltsanalyse nicht vorgegeben. Dies führt dazu, dass die Anzahl einiger Antworten innerhalb der Unterkategorien teilweise so gering ist, dass der Ausweis einer relativen Größe wenig aussagekräftig bleibt. ${ }^{28}$

\section{Kernergebnisse}

Die Aussagen der Interviewten lassen sich vier Themenbereichen zuordnen, auf die das Kategoriensystem aufbaut: Aussagen zum wirtschaftswissenschaftlichen Bezug in der aktuellen Tätigkeit, generelle Aussagen zum Studiengang Wirtschaftsrecht, Aussagen zu den wirtschaftswissenschaftlichen Kursen im Studiengang sowie Aussagen zu hilfreichen wirtschaftswissenschaftlichen Kompetenzen und Fachgebieten für Wirtschaftsjuristen.

\section{Aussagen zum wirtschaftswissenschaftlichen Bezug in der aktuellen Tätigkeit}

Knapp die Hälfte der befragten Absolventen arbeitet bei wirtschaftsnahen Arbeitgebern wie Finanzdienstleistern oder Industrieunternehmen (41\%). Es ist somit ein leichter Überhang klassisch juristischer Tätigkeitsfelder ${ }^{29}$ festzustellen (48\%). Weiterhin verbleiben einige der Ehemaligen an der Hochschule (11\%).

In ihrer aktuellen Tätigkeit beschäftigen sich die Befragten schwerpunktmäßig mit Verträgen sowie mit juristischer Beratung (55\%). Überwiegend kaufmännische Tätigkeiten $(13 \%)$ sind vor allem bei Juristen in leitenden Positionen festzustellen. Das Spektrum der Tätigkeitsfelder ist so vielfältig wie die unterschiedlichen Arbeitgeber,

28 Bspw. arbeiten zwei von 27 Interviewten zum Befragungszeitpunkt als Richter. Als Anteil ergibt sich hier eine relative Größe in Höhe von 7\%. Ob diese Größe einen Aussagegehalt hat, ist jedoch zweifelhaft, weshalb bei einigen der nun folgenden Ergebnisse ein zusammenfassender Anteil ausgewiesen wird, welcher die schwer zu kategorisierenden Antworten bündelt.

29 Tätigkeiten als Anwälte in Kanzleien und in Unternehmen. 
so dass sich bei den sonstigen Tätigkeiten (32\%) auch Aufgabenbereiche wie Bearbeitung von Insolvenzverfahren, Bankenaufsicht oder Geldwäscheüberwachung finden.

Es wurde weiterhin danach gefragt, mit welchen wirtschaftswissenschaftlichen Bereichen die Interviewten in ihrem Berufsalltag in Kontakt kommen. Hier nannte rund ein Drittel der Befragten wirtschaftswissenschaftliche Kennzahlen und Berechnungen (31\%), aber auch Buchhaltung und Bilanzierung wurden verhältnismäßig häufig erwähnt $(22 \%)$. Die Kommunikation mit Wirtschaftswissenschaftlern sowie die Anwendung von Fachsprache und Denkweise ist ebenfalls ein wichtiger Aspekt im $\mathrm{Zu}$ sammenhang mit wirtschaftswissenschaftlichen Aufgaben (18\%). ${ }^{30}$

Mehr als die Hälfte der Befragten arbeitet nach eigener Einschätzung weniger als $20 \%$ der Arbeitszeit an Aufgaben mit wirtschaftswissenschaftlichem Bezug (52\%). ${ }^{31}$ Der Großteil der von ihnen geleisteten Arbeit bezieht sich folglich auf klassisch juristische Aufgabenstellungen. Oft erfolgt dabei jedoch eine enge Zusammenarbeit mit Wirtschaftswissenschaftlern.

\section{Generelle Aussagen zum Studiengang}

Auch wenn der zusätzliche Abschluss einen wichtigen Grund für die Studienwahl darstellt (25\%), steht der Erwerb zusätzlichen Wissens im Vordergrund. Über den Nutzen des zusätzlichen Titels „LL.M.“ als Vorteil auf dem Arbeitsmarkt herrscht eine uneinheitliche Meinung. Einige Alumni sehen den Titel als Vorteil bei potenziellen Arbeitgebern, wohingegen andere einen ausländischen Abschluss bevorzugt hätten. ${ }^{32}$ Obwohl der Erwerb wirtschaftswissenschaftlicher Kenntnisse eine Hauptmotivation für das Studium darstellt (28\%), möchten viele Studierende auch ihr juristisches Fachwissen erweitern (13\%). Wenige haben den klaren Wunsch, im Unternehmen oder einer Wirtschaftskanzlei zu arbeiten (13\%). Vereinzelt genannte, weitere Gründe für die Studienwahl waren Überbrückung der Wartezeit bis zum Referendariat, der Standort Köln, Zufall oder die Erweiterung des eigenen Netzwerkes. ${ }^{33}$

30 Der restliche Anteil in Höhe von $29 \%$ setzt sich aus vielfältigen Aussagen zusammen, so z.B. Personalplanung oder Risikomanagement.

$3126 \%$ arbeiten nach eigener Einschätzung zwischen 20\% und 50\% ihrer Arbeitszeit an Aufgaben mit wirtschaftswissenschaftlichem Bezug, 22\% der Absolventen verbringen mehr als 50\% ihrer Arbeitszeit mit wirtschaftswissenschaftlichen Aufgaben.

32 Siehe dazu auch den LL.M.-Report des Anwaltsblattes: „Wird der LL.M. im Ausland erworben, so gilt er als Garant für gute Fremdsprachenkenntnisse, Erfahrung mit anderen Kulturen und Vertrautheit mit fremden Rechtssystemen. Dagegen ist das deutsche Pendant nichts wert, so scheint es. Es ist schließlich kein besonderer Verdienst, an seiner Heimatuniversität noch ein paar zusätzliche Kurse zu belegen, zum Teil sogar unter Anrechnung bereits erbrachter Studienleistungen. Es mag Studenten geben, die nur den schnellen Titel wollen, ja. Doch sie dürften nicht die Regel sein. Praxisnähe und Spezialisierung sind die Zauberworte, mit denen deutsche Universitäten für ihre Programme werben. “ Bubrowski, in: Anwaltsblatt Karriere, 2/2088, S. 87 ff.

33 Diese Aussagen erklären den verbleibenden Anteil in Höhe von 21\%. 
Weitere generelle Aussagen zum Studiengang betreffen die Studienstruktur, Studieninhalte über die Wirtschaftswissenschaften hinaus (bspw. englischsprachige Veranstaltungen) oder die Vermarktung des Studiengangs. Da diese Aussagen nicht für die wirtschaftswissenschaftliche Curriculumentwicklung relevant sind, werden sie an dieser Stelle außer Acht gelassen.

\section{Aussagen zu den wirtschaftswissenschaftlichen Kursen}

Die meisten Absolventen belegten die Veranstaltungen mit dem Anspruch, einen Überblick über die Wirtschaftswissenschaften zu erlangen sowie Begriffe zu lernen $(40 \%)$. Häufiger werden auch die Ziele genannt, Unterschiede zur juristischen Denkweise (13\%) oder Praxisbezüge (13\%) aufgezeigt zu bekommen. Weitere Ansprüche an die Veranstaltungen betreffen die Vorbereitung auf eine Selbstständigkeit oder so viel wie möglich zu lernen, um sich von anderen Juristen auf dem Arbeitsmarkt abzuheben. Manche Studierende gingen ohne Erwartungen in die Veranstaltungen. Andere geben offen zu, dass sie möglichst schnell und unkompliziert die „Scheine“ für die wirtschaftswissenschaftlichen Veranstaltungen erwerben wollten. ${ }^{34}$

Weitere Anmerkungen zu den wirtschaftswissenschaftlichen Kursen betreffen strukturelle und organisatorische Aspekte, die im Hinblick auf die inhaltliche Gestaltung des Lehrplans nicht von Bedeutung sind.

\section{Aussagen zu hilfreichen wirtschaftswissenschaftlichen Kompetenzen und Fachgebieten für Juristen}

Nach Meinung der Befragten sollten drei wirtschaftswissenschaftliche Kompetenzen im Studium erlernt werden, die im Berufsalltag zum Einsatz kommen:

- Wirtschaftswissenschaftliche Denkweise verstehen (34\%)

- Wirtschaftswissenschaftliche Fachsprache sprechen (34\%)

- Zahlen erfassen und Berechnungen durchführen (32\%)

Bei der Nennung konkreter Inhalte überwiegen die betriebswirtschaftlichen Themen. Am häufigsten wird das externe Rechnungswesen (23\%) erwähnt. Zudem beziehen sich die Aussagen des Öfteren auf Buchführung bzw. Buchhaltung (11\%) oder die Erstellung von Businessplänen (11\%). Die weiteren Aussagen zu relevanten betriebswirtschaftlichen Fachgebieten sind breit und über sämtliche Funktionsbereiche der BWL gestreut (41\%).

Die Bedeutung von Inhalten der VWL für Juristen beschränkt sich auf Themen, die mit speziellen Rechtsgebieten und damit speziellen Tätigkeitsfeldern zusammenhängen. Es beziehen sich insgesamt lediglich 14\% der Aussagen auf volkswirtschaftliche Fachgebiete.

34 Diese erneut schwer zu kategorisierenden Aussagen lassen sich zu einem Anteil von 34\% zusammenfassen. 


\section{E. Implikationen}

Interdisziplinäre Kommunikation ist für die Befragten notwendiger als die Nutzung von konkretem, wirtschaftswissenschaftlichem Fachwissen. Dies ist für das Curriculum und die Auswahl von Lehr-/Lerninhalten insofern relevant, als dass in den betriebs- und volkswirtschaftlichen Einführungsveranstaltungen lediglich ein Überblick über Grundbegriffe und Forschungsansätze der Wirtschaftswissenschaften gegeben wird. In der Betriebswirtschaftslehre sollte gemäß einer Gliederung nach Funktionen ${ }^{35}$ der Fokus weniger auf den Güterstrom und damit die Leistungserstellung und -verwertung (Beschaffung, Produktion, Absatz), sondern eher auf Geldströme bzw. unterstützende Funktionen (Rechnungswesen, Investition und Finanzierung) gelegt werden, da diese näher in beruflichen Lebenswelten wirtschaftsjuristischer Absolventen zu finden sind als klassisch-betriebswirtschaftliche Leistungserstellungsprozesse.

Was also sollten Wirtschaftsjuristen in BWL und VWL können? Die Antwort liegt eher in der Bewältigung von Handlungssituationen im Umgang mit Wirtschaftswissenschaftlern als in einem umfangreichen Wissenskatalog oder einem methodischen „Handwerkskoffer“. Im Berufsalltag geht es letztlich darum, Probleme nicht nur juristisch, sondern auch wirtschaftlich beurteilen zu können. Zweifelsohne ist dafür ein gewisses Repertoire von Begriffen ${ }^{36}$ notwendig: Es „bleibt bei jeder Art situativer Ziel- und Inhaltsbestimmung die Notwendigkeit, das zur Situationsbewältigung erforderliche Wissen (Begriffe, Strategien) in der kognitiven Struktur des Lernenden zu verankern." 37

Das notwendige Wissen und die notwendigen Begriffe zu vermitteln - das kann der Studiengang Wirtschaftsrecht in jedem Fall leisten, und das betriebs- und volkswirtschaftliche Curriculum kann inhaltlich am Bildungsbedarf der Zielgruppe ausgerichtet werden. Eine Übertragung auf Handlungssituationen erfolgt bisher lediglich durch einen interdisziplinären Diskurs mit den wirtschaftswissenschaftlichen Lehrenden. Hier wäre weiterführend zu überlegen, wie die Nutzung des Wissens zur Bewältigung von Handlungssituationen vertiefend in das Studium integriert werden kann.

\section{F. Ausblick}

Eine Bildungsbedarfsanalyse durch eine Absolventenbefragung durchzuführen, stellt eine mögliche Vorgehensweise zur Gestaltung von Studienangeboten dar. Hierbei sind die Erfahrungswerte der ehemaligen Studierenden berücksichtigt, um die Lehrpläne an den Bedürfnissen der Teilnehmer des Studiengangs anzupassen. Darüber hinaus kann eine weiterführende Befragung von Lehrenden, aktuellen Studierenden oder Arbeitgebern in Betracht gezogen werden, um einen breiteren Einblick zu er-

35 Zum Beispiel in Wöhe/Döring, Einführung in die allgemeine Betriebswirtschaftslehre, S. $43 \mathrm{f}$.

36 Hier wurden beispielsweise Opportunitätskosten, Grenznutzen, Deckungsbeitrag oder Break Even von den Absolventen genannt.

37 Erneut Reetz, Wirtschaftsdidaktik, S. 106. 
halten und verschiedene Perspektiven auf den Bildungsbedarf zu erfassen. Über die Befragung von Lehrenden können auch das Wissenschafts- und das Persönlichkeitsprinzip stärker in die Analyse eingebracht werden. Des Weiteren lässt sich die Bildungsbedarfsanalyse durch eine Marktbeobachtung ergänzen, indem beispielsweise Stellenanzeigen für (Wirtschafts-)Juristen analysiert und/oder ähnliche Studiengänge an anderen Hochschulen als „Benchmarks“ betrachtet werden.

\section{Literaturverzeichnis}

Bubrowski, Helene, LL.M. Einjähriges Auslandsstudium als Karriereschub? Teil 4: Deutschland. In: Anwaltsblatt Karriere, 2/2008, S. 87-89.

Böhm-Kaspar, Oliver/Schuchart, Claudia/Weishaupt, Horst, Quantitative Methoden in der Erziehungswissenschaft, Darmstadt 2009.

Euler, Dieter/Hahn, Angelika, Wirtschaftsdidaktik, 2. Auflage, Bern (u.a.) 2007.

Hameyer, Uwe, Systematisierung von Curriculumtheorien; in: Hameyer/Frey/Haft (Hrsg.), Handbuch der Curriculumforschung, Übersichten zur Forschung 1970-1981, Weinheim 1983, S. 53-100.

Kromrey, Helmut/Strübing, Jörg, Empirische Sozialforschung, Modelle und Methoden der standardisierten Datenerhebung und Datenauswertung, 12. Auflage, Stuttgart 2009.

Müller, Ulrich, Bildungsmanagement - Skizze zu einem orientierenden Rahmenmodell, in: Schweizer/Iberer/Keller (Hrsg.), Lernen am Unterschied, Bildungsprozesse gestalten, Innovationen vorantreiben, Bielefeld 2007, S. 99-121.

Reetz, Lothar, Wirtschaftsdidaktik, Eine Einführung in Theorie und Praxis wirtschaftsberuflicher Curriculumentwicklung und Unterrichtsgestaltung, Bad Heilbrunn 1984.

Schaper, Nicolas, Fachgutachten zur Kompetenzorientierung in Studium und Lehre, Unter Mitarbeit von Oliver Reis, Johannes Wildt, Eva Horvath und Elena Bender, Hochschulrektorenkonferenz, Projekt nexus 2012, http://www.hrk nexus.de/material/links/kompetenzorientierung/ (13.12.2013).

Schnell, Rainer/Hill, Paul B./Esser, Elke, Methoden der empirischen Sozialforschung, 7. Auflage, München 2005.

Teichler, Ulrich, Der Jargon der Nützlichkeit, Zur Employability-Diskussion im Bologna-Prozess 2008, in: Das Hochschulwesen 56 (3), S. 68-79.

Wöhe, Günter/Döring, Ulrich, Einführung in die allgemeine Betriebswirtschaftslehre, 24. Auflage, München 2010. 